\title{
La inclusión de los territorios mediante la apuesta por el emprendimiento sostenible: lo que implica, lo que está en juego y los obstáculos que se enfrentan
}

\author{
Fernando Luis Castellanos Obregón, PhD
}

\section{Resumen:}

La pretensión de promover emprendimientos sostenibles se enfrenta a circunstancias de índole económica, política, socio ambiental y sociocultural que se considera obstaculizan tal propósito.

Interesan particularmente las circunstancias socioeconómicas, políticas y culturales, porque se estima que son históricamente relevantes en el desencadenamiento de procesos combinados que podrían constituirse en externalidades negativas obstaculizadoras del empeño emprendedor en territorios emergentes de menor desarrollo relativo.

Aquí se examinan esos aspectos, en búsqueda de una salida.

Palabras clave: territorio, sustentabilidad, sostenibilidad, emprendimiento sostenible, migración, precariedad laboral.

The inclusion of territories through a commitment to sustainable entrepreneurship: what it implies, what is at stake and the obstacles they face

\begin{abstract}
The claim to promote sustainable ventures faces economic, political, socio-environmental and socio-cultural circumstances that are considered to hinder such purpose. Socioeconomic, political and cultural circumstances are of particular interest, because they are considered to be historically relevant in the unleashing of combined processes that could become negative externalities that hinder entrepreneurial endeavor in emerging territories with less relative development.
\end{abstract}

Keywords: territory, sustainability, sustainability, sustainable entrepreneurship, migration, job insecurity.

\section{Introducción}

"Los mercados no existen, se hacen. Su funcionamiento depende de la definición previa de las relaciones de propiedad, las normas impuestas por los gobiernos y los organizadores del Mercado mismo, las tradiciones y comportamientos de los participantes, y la tecnología concreta usada para realizar las transacciones. Todos estos aspectos determinan hasta qué punto el funcionamiento de un sistema específico de mercado es compatible con el desarrollo sostenible. Se trata, por lo tanto, de introducir reformas en los sistemas, con el propósito de asegurar que ambos sean compatibles".

Trigo y KaimowitzEconomía y sostenibilidad ¿pueden compartir el planeta? IICA, San José de Costa Rica, 1994. También alojado en FAO-CEDAF- Centro para el Desarrollo Agropecuario y Forestal, 1996.

Si bien, se vislumbra una luz para el emprendimiento sostenible en la iniciativa y el esfuerzo conjunto público-privado, de tal manera que si se intenta concretarlos en políticas públicas de juventud que retengan a los jóvenes y/o motiven a los que retornan, podrían obtenerse algunos logros; sin embargo, en territorios regionales de limitado desarrollo local, caracterizados, por lo general, por una fuerte vocación migratoria ${ }^{\mathrm{i}}$, y el escepticismo y desesperanza que tienen sus jóvenes,entre otras razones por la falta de oportunidades, resulta todo un desafío impulsar allí el espíritu empresarial, y más aún, el emprendimiento sostenible.

El primer paso para abordar el asunto a tratar, apunta a la conceptualización rigurosa y el planteamiento del problema de indagación, toda vez que la investigación social no sólo parte, sino que consiste, básicamente, en plantearse y enfrentar problemas que se derivan de lagunas, inconsistencias o deducción de consecuencias. Ahora bien, independientemente de su punto de origen, el tratamiento de un problema de investigación, cualesquiera que este sea, requiere de la precisión conceptual referida al asunto específico que se va a abordar. De otra parte, digamos todavía que, a un nivel más concreto, el proceso de indagación comprende la ubicación del problema en una determinada situación histórico-social y sus relaciones con otros asuntos. En el caso que nos ocupa de una apuesta por el emprendimiento sostenible y lo que conlleva, se hace necesaria, entonces, la fundamentación conceptual, la problematización, y el enmarcamiento contextual de la problemática a tratar.

*Notas al final. 
Metodológicamente, en primera instancia se abocó la indagación de las diferentes situaciones locales y atributos diferenciados, de losterritorios escogidos para configurar un estudio de caso. Para ello, se utilizó el análisis de entorno (environmentalscanning) a partir de su discusión y selección en jornadas de trabajo de campo y observación en las distintas comunidades y municipios; y también, mediante grupos focales y conversaciones con personas conocedoras de dichos territorios; para luego, efectuar su análisis mediante el método de configuraciones ${ }^{\text {ii }}$, propuesto por Ragin(1994)) el cual se concentra en las combinaciones de dicotomías presencia-ausencia (lo cual significa que las condiciones causales y los resultados están o bien presentes [1] o bien ausentes [0] en cada caso) y que a su vez brinda elementos de equiparación. Todo ello contribuyó metodológicamente, por una parte, a resaltar la diferencia (las particularidades de lo que allí ocurre) y, por otra parte, a vislumbrar las posibilidades que dichas zonas tienen para el desarrollo del emprendimiento sostenible. Por último, ese proceso también contribuyó a buscar salidas, para lo cual, complementariamente, fue de mucha ayuda la construcción de escenarios prospectivos a partir de opinión experta, los cuales coadyuvarán a trazar sendas, derroteros y trayectorias de desarrollo.

\section{Conceptualización y planteamiento del problema de indagación.}

Cualquiera que sea el punto de origen de una indagación, el tratamiento del problema allí planteado requiere en primera instancia de una delimitación conceptual, referida al asunto específico que se va a abordar. Siendo así las cosas se aborda, en primer lugar, el concepto de emprendimiento; para luego, esclarecer la diferencia entre sustentabilidad y sostenibilidad y con esos insumos, por último, abordar el concepto de emprendimiento sostenible, con todo y sus exigencias e incidencias. Si bien sobre este campo, práctica y conceptualmente se ha trabajado poco en L. A., y particularmente en Colombia, hay que señalar que ya hay una experticia acumulada y se ha llegado a unos acuerdos mínimos en torno a su ejercicio. Si bien convencionalmente se entiende el emprendimiento como creación de riqueza nueva incremental (Exedu,1991) el enfoque de las externalidades, que Coase plantea en La empresa, el mercado y la ley (1960) permite visualizar la relación y efecto del emprendimiento ${ }^{\mathrm{iii}}$ en los ámbitos, no sólo económico sino medioambiental y social (Rodríguez, D., 2016:3).

De ahí que, en gracia de discusión, como siguiente paso se establece conceptualmente la diferencia entre sustentabilidad y sostenibilidad; para luego, abordar el concepto de emprendimiento sostenible.

Respecto a los dos conceptos en cuestión: sustentabilidad y sostenibilidad, de acuerdo con las Naciones Unidas la diferencia conceptual radica en que lo sustentable se refiere al proceso por el cual se preservan, conservan y protegen sólo los recursos naturales, para el beneficio de las generaciones presentes y futuras.

Por ello, desde el punto de vista de la sustentabilidad no se toman en cuenta las necesidades sociales, políticas ni culturales del ser humano; mientras que, por el contrario, el enfoque de sostenibilidad trata de satisfacer tanto las necesidades económicas como las sociales, de diversidad cultural y de un medio ambiente sano de la actual generación, pero sin poner en riesgo la satisfacción de las mismas a las generaciones futuras.

Según Pacheco et alia (2010) señalan "la sostenibilidad busca lograr la interacción a largo plazo entre sociedad y medio ambiente, lo cual implica el bienestar social y ambiental" (Pacheco et alia, 2010).

Ahora, como según Crals y Vereeck (2005) por definición la sostenibilidad está orientada al largo plazo "entra en conflicto con las visiones de corto plazo y los lineamientos miopes de política pública"(Crals\&Vereeck, 2005)los cuales no tienen en cuenta el conjunto de circunstancias que rodean una situación, y sin las cuales ésta no se puede comprender correctamente ${ }^{\text {iv }}$.

Ahora que ya hemos precisado y nos queda claro lo qué lo sostenible significa, lo cual para efecto de lo que nos proponemos es muy importante, se ligan los conceptos pertinentes y se pasa a examinar el complejo concepto de emprendimiento sostenible $\mathrm{v}$.

Conceptualmente, el emprendimiento sostenible, además de su obvio componente económico, que como ya se mencionó, lo caracteriza por definición como creación de riqueza nueva incremental (Exedu, Ibid.), implica también la protección del medio ambiente circundante y el desarrollo social en el entorno en el cual se ubica.

Para Patzelt et alia (2011) el emprendimiento sostenible abarca el emprendimiento ecológico (ecoemprendimiento); el emprendimiento social, definido como "la visión innovadora, el espíritu transformador y la pasión por el hacer, capaces ambos de generar espacios donde puedan canalizarse desde la demanda de más y mejor democracia real, hasta el clamor por el cuidado del planeta y el acceso a condiciones de vida dignas para millones de personas que padecen hambre, pobreza y exclusión" (Barlocci, 2011); y el emprendimiento convencional o propiamente empresarial (Patzelt et alia, 2011, Ibid.). 
En lo social, el emprendimiento sostenible contempla "aspectos sociales y éticos relacionados con el talento y los derechos de los trabajadores, el trabajo infantil, la inclusión de género, y la participación de los trabajadores en los beneficios" (Crals\&Vereeck, 2005, Ibid.).

En lo medioambiental, contempla producción limpia, diseño y acciones respetuosas con el medio ambiente.

Por último, vela por la solvente supervivencia financiera de la empresa (Crals\&Vereeck, 2005, Ibid.).

Ahora bien, generalmente se considera que la actividad emprendedora dinamiza un mayor crecimiento económico y, por consiguiente, el desarrollo ${ }^{\mathrm{vi}}$ y bienestar social.

No obstante, Álvarez et alia (2014) señalan que a más de que no siempre es realizable, no siempre la actividad empresarial genera el suficiente crecimiento capaz de alcanzar desarrollo y bienestar para las comunidades locales (llamadas también regionales, territoriales y/o de provincia) sobre todo, en contextos emergentes y de pobreza extrema.

Eso ocurre, por regla general, porque si bien el desarrollo territorial parte del crecimiento económico, constituye ante todo un proceso socioeconómico, cultural, tecnológico, político y ambiental complejo, que exige el reenfoque y la reorientación de los esfuerzos y los recursos hacia un proyecto compartido de región. En esa medida, pasa también por la dotación de factores tanto naturales como creados; y está determinado por las relaciones de poder imperantes; por los conflictos que suelen caracterizar a las sociedades de nuestros países, particularmente las de provincia; y por la capacidad de los dirigentes para tomar las decisiones necesarias y asumir la responsabilidad de ejecutar la gestión ${ }^{\text {vii }}$ que desaten la atractividad ${ }^{\text {viii }}$ de la región y potencie su competitividad sistémica y su desarrollo ${ }^{\text {ix }}$.

En el contexto de la Provincia de Ubaté, caso seleccionado como objeto de estudio e indagaciónLas preguntas que problematizan ${ }^{\mathrm{x}}$ el emprendimiento sostenible, y que guiaron la pesquisa, son las siguientes: ¿Por qué en un circuito con tradicional vocación migratoria y desesperanza de los jóvenes, como es el de la Provincia de Ubaté (Departamento de Cundinamarca, Colombia) es difícil apostar por el emprendimiento sostenible? ¿Bajo el supuesto de que hay condiciones para ello, qué tan significativas y qué efecto tienen las manifestaciones de recelo e incredulidad y la propensión a la emigración por parte de los jóvenes respecto al propósito de promover el emprendimiento sostenible? ¿Cómo enfrentar el reto?

\section{Fundamentación y enmarcamiento contextual de la problemática en la Provincia De Ubaté.}

A un nivel ya más específico, este apartado comprende la ubicación del problema que implica apostar por el emprendimiento sostenible en una determinada situación histórico-social, y sus relaciones con otros fenómenos. A continuación, entonces se hace necesaria la fundamentación y enmarcamiento contextual en la Provincia de Ubatéde la problemática formulada.

Es evidente que Colombia atraviesa en la actualidad por una situación particularmente conflictiva, agudizada el último año por procesos socio-políticos y económicos que, ligados con dificultades de crecimiento, inestabilidad social y una inequitativa distribución del ingreso, han exacerbado las tensiones y pugnas entre clases, y evidenciado una crisis generalizada de valores que impacta particularmente a los jóvenes.

De una parte, la forzada migración originada por la violencia en los territorios locales ha propiciado el desarraigo de importantes masas de población.

A ese proceso se suma, por lo general, la emigración de jóvenes en búsqueda de mejores alternativas de estudio que les posibilite la oportunidad de conseguir empleos mejor remunerados.

Por otro lado, el desordenado y salvaje crecimiento de las ciudades, y la desintegración del núcleo familiar, han contribuido a desdibujar valores tales como el amor al estudio y al trabajo, la ética del esfuerzo propio, la honestidad, el apego a la familia, el ingenio y capacidad emprendedora; y han potenciado la desesperanza, confusión y disminución de la salud mental, dándole paso a nuevos patrones de comportamiento muchas veces asociales, como el aislamiento social, la depresión, recelo, incredulidad, desmotivación, desesperanza y tendencias suicidas.

Si tenemos presente que según Kliksberg (2007) los jóvenes latinoamericanos son cerca del $40 \%$ de la población de la región. Y, por otro lado, que según estimativos proyectados a partir del último censo, los menores de 18 años representan el 30,6\% de la población de la Provincia de Ubaté, podemos colegir que para los gobiernos la situación de los jóvenes, que se enfrentan a la cruda realidad de la falta de oportunidades, es preocupante, no sólo por su peso cuantitativo específico en los estimativos demográficos, sino porque se considera que los jóvenes son el mayor agente de cambio potencial, toda vez que se supone tienen, según los especialistas, una facilidad especial para el manejo de nuevas tecnologías, al mismo tiempo que una más alta disposición que cualquier otro sector social a comprometerse con ideales, con retos colectivos, y tristemente no hay políticas públicas claras y precisas que les brinden una salida. 
Proponer, entonces, el desarrollo de emprendimiento sostenible, a más de que no es fácil de lograr, no es tampoco la panacea para el desarrollo local, y menos en territorios como el de la Provincia de Ubaté, de pobreza creciente, crecimiento reducido, inercia generalizada y poca claridad sobre el rumbo que se le quiere dar a esta juventud recelosa e incrédula.

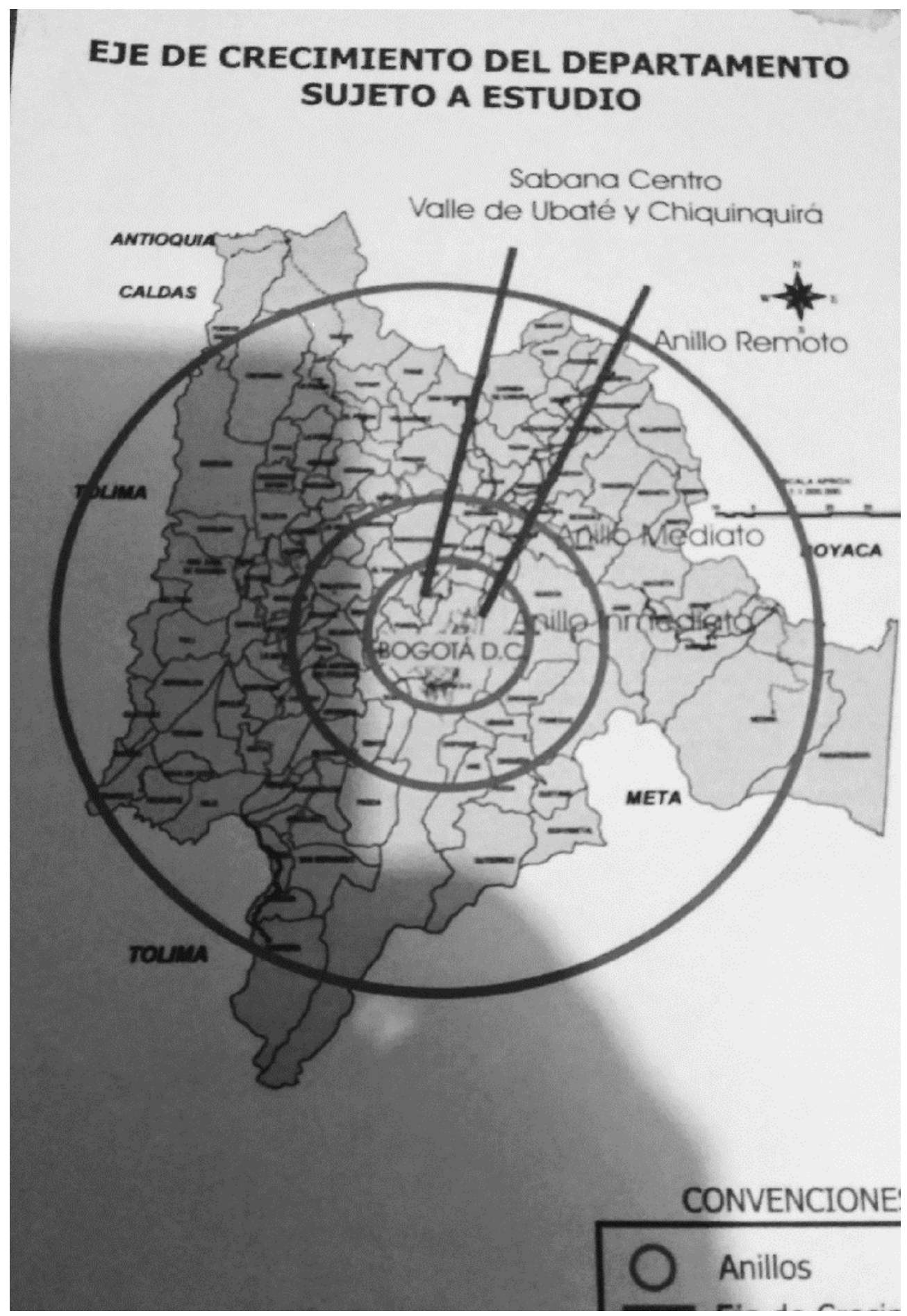

FUENTE: Obsoged/Laso elaborado por Fernando Castellanos y otros, para uso académico e investigativo.

Ahora bien, se vislumbra una luz para el emprendimiento en la iniciativa y el esfuerzo conjunto público-privado; así que si se intenta concretar los esfuerzos en políticas públicas de juventud, que retengan a los jóvenes y/o motiven a los que retornan, podrían obtenerse algunos logros. 
Sin embargo, en un territorio de limitado desarrollo local como lo es el de la Provincia de Ubaté, a 90 klms de Bogotá en el Centro de Colombia, caracterizado por una fuerte vocación migratoria y la desesperanza y el escepticismo que tienen sus jóvenes por la falta de oportunidades en sus territorios, entre otras razones; resulta todo un desafío impulsar el espíritu empresarial, y más aún, el emprendimiento sostenible, toda vez que la migración por mejores oportunidades de estudio y trabajo, se comporta en forma análoga a la movilidad del capital: mientras este tiende a moverse desde localizaciones con bajas tasas de rendimiento hacia aquellas con altas tasas; análogamente, sobre todo los jóvenes tienden a migrar desde economías con educación de baja calidad y empleo precario remunerado con bajos salarios (territorios pobres como los municipios de la Provincia de Ubaté) hacia las zonas que ofrecen mejor educación y remuneraciones más altas (Bogotá y el extranjero). Desde nuestra óptica, tales condiciones reinantes de indolencia, apatía, incertidumbre y desesperanza, que impactan en especial a las nuevas generaciones, exigen el lineamiento de acciones que revaloricen su dimensión de futuro.

\section{Desarrollo del estudio de componentes, mecanismos y procesos concretos históricamente determinados en la Provincia De Ubaté.}

\section{A. Indagación de las diferentes situaciones locales en la Provincia de Ubaté.}

Como ya se adelantó, se hace uso, en primer lugar, del análisis de entorno (environmentalscanning) que es una herramienta de planificación y gestión estratégica y prospectiva "que permite realizar un examen temprano de contexto $^{x i}$, que da cuenta tanto de las condiciones como de los factores de cambio de desarrollo que tiene un determinado territorio" [Nanus B.(1982); Neufeld, W.P. (1985)] Analizada detenidamente la definición de contexto, es posible percibir implícitamente que el sitio desde donde uno se sitúa para efectos de cualquier análisis, resulta afectado positiva o negativamente por procesos y/o centros de poder y de decisión más o menos ajenos a nuestro control.

Sí lo específico de un territorio son los procesos culturales, económicos y políticos concretos que allí acontecen, como resultado de las relaciones entre actividades en las cuales la población participa es, entonces, la identificación de esos procesos y su análisis lo que constituye el diagnóstico básico de ese territorio.

En Cundinamarca las diferencias territoriales son notorias, no solamente en términos físico-geográficos, sino en su configuración histórica, las cuales reflejan formaciones sociales, estructuras políticas y procesos de desarrollo diferenciados en y entre todos ellos. La Provincia de Ubaté no es la excepción. Conformada por los municipios de Tausa, Sutatausa, Cucunubá, Carupa, Lenguazaque, Guachetá, Fúquene, Susa, Simijaca y Ubaté, está ubicada al nororiente del Departamento de Cundinamarca.

Predomina allí la población rural, la cual según cálculos basados en el último censo corresponde al $61 \%$ de la población total de la Provincia ${ }^{x i i}$. Esta circunstancia, de alguna manera signa su vocación extractiva y agropecuaria y su potencial agroindustrial. Con distinto grado y condiciones de desarrollo relativo, dentro de las actividades empresariales que allí se llevan a cabo, predomina la producción de derivados lácteos. Eso a pesar de que la leche está perdiendo competitividad. Principalmente esto ocurre por las fluctuaciones de sus precios, debido básicamente a los altos costos de la explotación ganadera, que están volviendo la leche inaccesible para los pequeños productores y redundan en detrimento de la calidad de la leche misma, y por ende de los derivados que allí se producen.Otras actividades manufactureras regionales, de corte más tradicional, son la artesanía de tejidos de lana, curtiembres y los chircales, en los que se producen artesanalmente ladrillos, tejas y adobes. La región cuenta también con unos sectores minero y comercial que, aunque estructurados de manera tradicional, son básicos para su economía.

No obstante, con una economía regional predominantemente de subsistencia, prácticamente cerrada,y con la producción agrícola estancada, se potencia la minería compuesta de pequeños explotadores y da lugar al fortalecimiento de la actividad comercial. La mayoría de estas actividades operan básicamente dentro del mercado regional, y sobre todo la minería, curtiembres y los chircales son altamente contaminantes. Un análisis detallado de la estructura etaria de la Provincia de Ubaté, permite concluir que aunque la mayor parte (49\%) de sus habitantes se concentran en el segmento de 0 a 25 años, la Provincia ha perdido paulatinamente buena parte de su fuerza laboral (población comprendida en el rango de edad entre los 15 y los 65 años). Ello pone en evidencia, tal como lo mencionan diferentes agentes sociales regionales y algunos expertos (como es el caso de los investigadores del Cepec de la U. del Rosario), el hecho de que en la región se registran numerosas migraciones de personas, en su mayoría jóvenes, en busca de mejores oportunidades (principalmente hacia Bogotá). De acuerdo con el Cepec "lo anterior confirma el reto que enfrenta la Provincia de Ubaté respecto a la formulación de una agenda de proyectos económicos que, además de crear dinámicas de generación de valor, brinden oportunidades laborales de calidad para sus habitantes". De otro lado, también es importante mencionar que más de la mitad de la población de la Provincia de Ubaté está concentrada en el área rural (59\%), tendencia que corresponde a una estructura productiva concentrada en actividades agrícolas, pecuarias y mineras. 
Respecto a las características idiosincráticas de los pobladores de esta región, básicamente las actividades que lleva a cabo la gente giran en torno a la posibilidad de conseguir un empleo, de obtener un ingreso decoroso, de mejorar sus condiciones de vida. En ese sentido, la gente mira quién puede hacer algo por ellos ¿Los políticos? ¿La empresa privada? ¿El Estado? En consecuencia, sopesan sus actuaciones y las oportunidades que se les brindan, y participan o se marginan escépticos. Es, como puede verse, una perspectiva personal basada en lealtades relativas que no requieren ninguna creencia en las cualidades del patrón o del gobernante, sino que se relacionan más con la recompensa e incentivos materiales.

B. Análisis de las particularidades de lo que ocurre en la Provincia de Ubaté mediante dicotomías presenciaausencia de atributos diferenciados y/o elementos de equiparación

TABLA 1 Provincia de Ubaté: dicotomias presencia-ausencia de atributos diferenciados y elementos de equiparación

\begin{tabular}{|c|c|c|c|}
\hline $\begin{array}{l}\text { Dicotomías } \\
\text { Items }\end{array}$ & $\begin{array}{l}\text { Presencia } \\
\text { (1) }\end{array}$ & $\begin{array}{l}\text { Ausencia } \\
(0)\end{array}$ & Observación \\
\hline 1. Hay creación de riqueza nueva incremental & & 0 & \\
\hline 2. El emprendimiento es un tema sólo de la agenda académica & 1 & & \\
\hline $\begin{array}{l}\text { 3. El emprendimiento es un tema de la agenda pública } \\
\text { territorial }\end{array}$ & & 0 & \\
\hline 4. Se discuten iniciativas empresariales & & 0 & \\
\hline 5.Se atraen nuevas inversiones y tecnologías & & 0 & \\
\hline 6.Alto grado de emprendimiento innovador & & 0 & \\
\hline $\begin{array}{l}\text { 7.Hay nuevos emprendimientos que generan buen empleo e } \\
\text { ingresos competitivos }\end{array}$ & & 0 & \\
\hline $\begin{array}{l}\text { 8.Los emprendimientos existentes no explotan los recursos } \\
\text { naturales }\end{array}$ & & 0 & \\
\hline 9.Las empresas contribuyen a la degradación ambiental & 1 & & \\
\hline $\begin{array}{l}\text { 10.En el ámbito económico-social se observa desigual } \\
\text { distribución del Ingreso }\end{array}$ & 1 & & \\
\hline 11.Hay concentración de la tierra & 1 & & \\
\hline 12.Se afronta la lucha contra la pobreza & & 0 & \\
\hline 13.Las empresas establecen un equilibrio con su entorno & & 0 & \\
\hline 14.Hay precariedad Laboral & 1 & & \\
\hline 15.Hay difíciles condiciones en el mercado de trabajo & 1 & & \\
\hline $\begin{array}{l}\text { 16.Los jóvenes no cuentan con red de relaciones sociales, es } \\
\text { decir, capital social que puedan impulsarlos }\end{array}$ & 1 & & \\
\hline 17.La precarización laboral afecta sobre todo de los jóvenes & 1 & & \\
\hline 18.Hay falta de oportunidades & 1 & & \\
\hline 19. Hay desesperanza en los jóvenes & 1 & & \\
\hline $\begin{array}{l}\text { 20.Hay crecimiento de los empleos precarios de baja } \\
\text { productividad y remuneración }\end{array}$ & 1 & & \\
\hline 21.Hay desempleo & 1 & & \\
\hline
\end{tabular}




\begin{tabular}{|c|c|c|}
\hline 22.Hay pobreza & 1 & \\
\hline $\begin{array}{l}\text { 23.Hay una verdadera explosión de desigualdad, exclusión y } \\
\text { vulnerabilidad social }\end{array}$ & 1 & \\
\hline $\begin{array}{l}\text { 24.Hay políticas que podrían mejorar estructuralmente la } \\
\text { situación }\end{array}$ & & 0 \\
\hline $\begin{array}{l}\text { 25.Habilidad del emprendedor para reconocer y aprovechar } \\
\text { oportunidades }\end{array}$ & & 0 \\
\hline $\begin{array}{l}\text { 26.Respeto por el medio ambiente y las disposiciones y } \\
\text { políticas ambientales }\end{array}$ & & 0 \\
\hline $\begin{array}{l}\text { 27.Reconocimiento del medio natural y comunitario por parte } \\
\text { de los emprendedores }\end{array}$ & & 0 \\
\hline 28.Desconfianza en las Instituciones & 1 & \\
\hline 29. Vocación migratoria sobre todo de los jóvenes & 1 & \\
\hline $\begin{array}{l}\text { 30.Movilidad de los/las jóvenes en búsqueda de mejores } \\
\text { oportunidades de estudio y laborales }\end{array}$ & 1 & \\
\hline 31.Localización Estratégica & 1 & \\
\hline 32.Alianzas Sector Público - Sector privado & & 0 \\
\hline 33.Indiferencia ciudadana ante la negligencia de los dirigentes & 1 & \\
\hline 34.Desidia & 1 & \\
\hline 35.Desafección & 1 & \\
\hline $\begin{array}{l}\text { 36.Indiferencia por lo que ocurre en el contexto cercano y en el } \\
\text { mundo y por lo que hacen los dirigentes }\end{array}$ & 1 & \\
\hline 37.Hay analfabetismo Funcional & 1 & \\
\hline 38.Falta de voluntad política y de diligencia de los dirigentes & 1 & \\
\hline 39.La gente de edad está más comprometida que los jóvenes & 1 & \\
\hline 40.El comportamiento cívico deja mucho que desear & 1 & \\
\hline $\begin{array}{l}\text { 41.Aumento de la delincuencia e inseguridad motivadas por } \\
\text { problemas sociales }\end{array}$ & 1 & \\
\hline 42.Aumento del desempleo y diferencias sociales muy notorias & 1 & \\
\hline
\end{tabular}

FUENTE: diseño y tabulación Fernando L. Castellanos Obregón investigador de OBSOGED/LASO. El número 1 indica la presencia de una condición y/o situación; a su vez, el 0 indica la ausencia de una condición y/o situación

Se presentan en la Tabla 1, los atributos diferenciados y las diferentes situaciones locales, los cuales se han discutido y seleccionado en jornadas de trabajo de campo y observación en los municipios, y mediante grupos focales y conversaciones con personas conocedoras de la Provincia y sus municipios; y se utiliza para el análisis pertinente el método propuesto por Ragin(1994)) de configuraciones; que se concentra en las combinaciones de dicotomías presencia-ausencia (lo cual significa que las condiciones causales están o bien presentes [1] o bien ausentes [0] en cada caso) y que a su vez brinda elementos de equiparación; todo lo cual contribuye, por un lado, a resaltar la diferencia (las 
particularidades de lo que ocurre en la Provincia de Ubaté) y por otra parte, a vislumbrar las posibilidades para el desarrollo del emprendimiento sostenible allí.

Como se desprende del análisis de la información consolidada en la tabla, en primer lugar, el emprendimiento no pasa de ser un asunto de la agenda académica sin que se refleje en la Provincia en creación efectiva de riqueza nueva incremental, ni en la discusión de iniciativas empresariales por parte de la comunidad y sus dirigentes, ni en la atracción hacia la región de nuevas inversiones y tecnologías que generen innovaciones y empleos con ingresos competitivos.

Para el desarrollo territorial de la Provincia, se enfrenta entonces, un complejo trilema entre cultura, tradición y tecnología

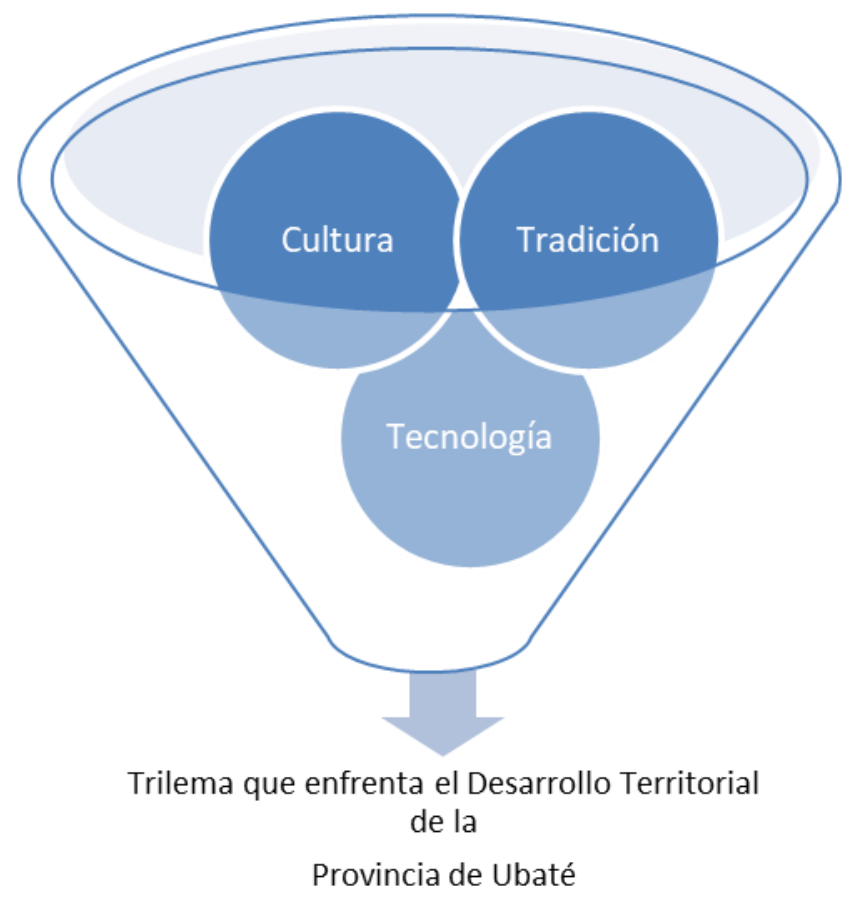

FUENTE: diseño Fernando L. Castellanos Obregón investigador de OBSOGED/LASO.

Por otra parte, hay concentración de la tierra; los emprendimientos existentes contribuyen a la degradación ambiental; hay, en general, difíciles condiciones en el trabajo y precariedad laboral con empleos de baja productividad y remuneración.

La precarización laboral afecta sobre todo a los jóvenes, que no cuentan con redes de relaciones sociales, es decir, capital social que pueda impulsarlos. Para los jóvenes migrantes el establecimiento de redes tiene una importancia crucial tanto en la localidad de origen como en la ciudad de destino, pues facilitan su experiencia migratoria. Las redes sociales son la principal ayuda a la que el joven migrante recurre, aún por encima de las instituciones académicas y/o gubernamentales.

Como hay desigualdad, desempleo y falta de oportunidades, por tanto, exclusión y vulnerabilidad social ${ }^{\text {xiii }}$, la desesperanza de los jóvenes respecto de un futuro mejor es grande. Todo ello les genera recelo y desconfianza en las instituciones, lo cual los incita a emigrar en búsqueda de mejores oportunidades de estudio y laborales.

$\mathrm{G}^{* *}$ tiene 33 años y dice ser una orgullosa "Susense" de nacimiento. Es enfermera y una emprendedora. Enfatiza que tiene una formación académica sólida, y además experiencia social y como empresaria en el Estado de Texas, en Estados Unidos. "Tengo experiencia empresarial y en ayuda social en otras naciones donde he vivido y en donde he podido trabajar en estos últimos 8 años" señala. Indica que tomó la decisión de regresar pues "junto con algunos de mis conciudadanos, decidimos participar para que cambiemos el destino en Susa"

Lo que más les molesta a los jóvenes es la desidia y negligencia de los dirigentes, quienes a pesar de la riqueza regional y de la localización estratégica de la Provincia, están sumidos en la inercia y la indecisión sobre el futuro Provincial. 
Así, la gente de edad está más comprometida que los jóvenes, muchos de los cuales se caracterizan por su indiferencia con lo que ocurre en el contexto cercano y en su entorno inmediato y por lo que hacen los dirigentes.

La gente de edad es más consciente a pesar del aumento de la inseguridad y de la constante desmejora de las condiciones de vida, particularmente debido a la falta de voluntad política y de diligencia de los dirigentes para llevar a cabo acciones efectivas que lo eviten.La búsqueda de objetivos ambiciosos por parte de los jóvenes estaría relacionada con la capacidad de asumir riesgos, como los asociados a la migración.

Las personas que tienden a emigrar, sobre todo las de provincia y áreas rurales, tienen índices mayores de insatisfacción en su vida, lo cual es lógico, si no, no se irían ${ }^{\text {xiv }}$. Muchas veces, la migración es más una solución que un problema, porque quien se marcha lo hace buscando oportunidades y una vida mejor. Pero la movilidad encierra, a su vez, un problema porque emigrar supone tensiones y dificultades.

$L^{* *}$ lo expresa así: "Me fui a vivir afuera, para estudiar. Pero por asuntos económicos tuve que retornar, ya que el costo de vida era muy alto. Al principio sentí frustración, porque anhelaba lograr una meta que me había trazado. Ahora tengo otras expectativas, y estoy resuelta a empezar una nueva vida para llevar a cabo mis planes, y así más adelante irme de nuevo a cumplir mis anhelos pero ya contando, esta vez si, con una red de apoyo."

En concreto, se está de acuerdo con Gómez (2015) cuando afirma que "la construcción de redes es un evento inevitable y necesario durante el proceso migratorio, hasta tal punto que puede decirse, inclusive, que es el punto neurálgico de este fenómeno, debido a que los afectos y lazos que se van entretejiendo configuran y definen el sentido que va tomando la migración, incluido el proceso de retorno (Gómez, 2015:50)

En la presentación del libro El desarraigo. La violencia del capitalismo en una sociedad rural (Bourdieu \&Sayad, 2017)Amín Pérez recalca que "fuera de su hábitat, en un estado de precarización social y espacial, pero sobre todo sin trabajo o en dificultades para adaptarse al nuevo entorno, la población rural queda atrapada en un día a día que le imposibilita vislumbrar claramente su futuro obligado a desapropiarse de la cultura que le pertenece y apropiarse de una nueva cultura urbana y distinta" (Pérez, 2017)

Las políticas de empleo sometidas al impacto de la mercantilización imperante que consideran el mercado como "estado de naturaleza", partiendo de la protección pública frente a la precariedad laboral y el desempleo, terminan por responsabilizar a los jóvenes de refugiarse en la dependencia familiar y estatal ${ }^{\mathrm{xv}}$. Es así que proponen el emprendimiento como escudo de salvación.

La verdad es que en el sustrato de las políticas propuestas de emprendimiento hay varias falacias. En primer lugar, el mercado dejó de ser entendido como el problema (causante del desempleo) a partir del trabajo por cuenta propia que se entronizó como único merecedor del apoyo del Estado. La protección colectiva de los derechos laborales se criminaliza como rigidez del mercado, para pasar a definir el empleo como una responsabilidad personal de cada individuo a partir de su iniciativa emprendedora. Con ello, el trabajo por cuenta ajena (empleo asalariado) queda devaluado como indigno de respaldo público.

\section{Construcción de escenarios Prospectivos para la Provincia de Ubaté, en la búsqueda de una posible salida.}

El análisis del entorno es un instrumento que permite evaluar la incertidumbre del contexto "que es generada por la impredictibilidad del comportamiento de los factores y, por los mismos cambios que se introducen o introducirán en un territorio, mediante proyectos" (Fernández Torres, A. OpCit consultada).

Esta herramienta del análisis del entorno, facilita la comprensión de diversos contextos (político, económico, jurídico, ambiental, social, cultural, educativo, tecnológico, etc.) en los que se desarrollará una acción estratégica en un determinado horizonte de tiempo.

Por esa capacidad heurística se utiliza también en procesos tanto de planificación y gestión estratégica como de diseño y construcción prospectiva de escenarios.

Se aboca, entonces en este apartado, la búsqueda de salidas.

Para ello se considera que, complementariamente al análisis de entorno, es de mucha ayuda la construcción de escenarios prospectivos, configurados a partir de opinión experta, toda vez que dicha complementación permite dotar de características al entorno, las cuales sirven de punto de partida a la construcción de imágenes de futuro, y coadyuvan a trazar sendas, derroteros y trayectorias futuras para la Provincia de Ubaté.

Es lo que a continuación se realiza en una prospección u horizonte de tiempo al año 2035.

Escenario Optimista: Como en las runas, todos a unas...

132 
Más identificados con la Provincia, a partir de su compromiso, responsabilidad social y liderazgo, los agentes sociales e instituciones públicas y organizaciones privadas y sociales han logrado la concertación y disposición conjunta y voluntaria para explorar las externalidades locales, aprovechar las ventajas del aprendizaje colectivo y organizar socialmente la coordinación económica y de apoyo institucional, para la ejecución de proyectos compartidos de emprendimiento sostenible.

De esa manera, se ha generado una mayor racionalidad territorial para la utilización de los recursos, el nivel de competitividad de la Provincia es más alto; y el incremento de las actividades productivas y de servicios la ha sacado del marasmo en que se encontraba y la han hecho menos vulnerable a las crisis.

Tales circunstancias han fortalecido las Pymes, que han iniciado algunas experiencias innovadoras exitosas, toda vez que la educación se ha convertido en prioridad y motivación para los jóvenes, en la medida en que la crisis fiscal del Estado lo ha permitido. Así, se han operado cambios en el patrón de especialización de la Provincia; y se ha generado una mayor conciencia sobre lo que hay que hacer para su desarrollo.

En la gestión territorial se ha logrado mayor coordinación y sinergia de las políticas públicas no sólo en el sector carbonífero, sino también en los dinámicos sectores de la producción láctea, comercio, transporte, ecoturismo y agroturismo, los cuales han propiciado la conformación de un corredor extractivo, un corredor agroindustrial, un corredor comercial y de logística, transporte y distribución y un atractivo corredor turístico.

\section{Escenario Pesimista: Fatalidad es nuestro sino y así mismo fatal es nuestro destino...}

Si bien la Provincia de Ubaté es el espacio físico y social de Cundinamarca y el centro del país con mayores condiciones estratégicas para el desarrollo, por su ubicación y la feracidad de sus tierras; por su cercanía a Bogotá, su mercado natural; y por su diversidad económica, social, cultural, política y medio ambiental que permite identificar zonas que como Simijaca y Susa a lo largo del valle, en la cuenca alta del Río Suárez en su nacimiento en la Laguna de Fúquene, propicias para el desarrollo productivo por sus condiciones y características, la marcada heterogeneidad social y de vocaciones, combinada con un crecimiento inequitativo de los municipios que conforman el territorio de la Provincia dificultan su acceso competitivo al mercado y son fuente de desigualdades y conflictos.

En consecuencia, resulta intrincado pensar la Provincia de Ubaté como una comunidad con una visión y unos intereses compartidos, en la que se pueda construir socialmente un futuro común en los próximos quince años.

En una situación tan ambigua y difícil o cuando menos complicada, tiende a aumentar la complejidad de la gestión de la Provincia; toda vez que al tiempo que disminuye la gobernanza también se dificulta la gobernabilidad y, sobre todo, la capacidad de coordinación de proyectos consensuados, se debilitan las instituciones y se desmoralizan los principales agentes sociales.

Por ello, a lo sumo se pueden explorar los senderos que se han de recorrer, para que los habitantes de la Provincia retomen su papel de ciudadanos conscientes e informados, y enderecen el rumbo de este territorio provincial tan rico en posibilidades y en talento humano.

\section{Escenario Posible: No hay nada que nos propongamos que no lo podamos lograr...}

A no dudarlo, el de hoy es un mundo de cambios cruciales que van en muchas direcciones: en las instituciones, en las comunicaciones y la información, en la ciencia, la técnica y la tecnología, en los mercados, en el medioambiente, en la gestión, en la mentalidad de la gente y en la vida cotidiana.

De seguir por el mismo rumbo que ha seguido, la Provincia de Ubaté aumentará su vulnerabilidad, y enfrentará el empeoramiento paulatino del ritmo de crecimiento de la producción y del empleo, lo cual, poco a poco, llevará a este territorio cundinamarqués a una situación cada día más difícil de manejar.

Sin embargo, dentro de tan crítica situación, existen territorialmente en la Provincia de Ubaté hechos portadores de futuro y áreas que inclinarán las fuerzas hacia un mejoramiento de la situación. Es así como, la decisiva capacidad de los diversos agentes sociales logrará realizar las escogencias estratégicas de derrotero y acción más convenientes, las cuales generarán una ruptura profunda con la tendencia de la Provincia al estancamiento y, por consiguiente, una renovación en la visión de los dirigentes y un cambio cultural de mentalidad, hacia el emprendimiento sostenible, con los esfuerzos articulados del sector público y del sector privado.

La cooperación, el talento humano y la construcción social de una identidad de territorio y de Provincia a partir de un proyecto compartido de desarrollo, ocuparán lugar prioritario, seguido de la renovación productiva, que dinamizará la economía provincial mediante la acentuación de sus atractivos para la inversión; promoverá el cambio técnico; recuperará la agroindustriapérdida; y conformará, de manera inteligente e informada, cadenas productivas estratégicas 
y sostenibles, mediante la potenciación de las vocaciones carbonífera, agrícola y lechera de la provincia y la sabia diversificación hacia el agroturismo y el ecoturismo.

También, en un horizonte de quince años, se habrá logrado un vuelco institucional, el cual se manifestará en el logro de un mejor y sólido sistema educativo en la Provincia, pertinente y capaz de fomentar el conocimiento y la innovación; y que igualmente, formará en la ética del emprendimiento sostenible y la solidaridad social, y permitirá a la Provincia de Ubaté avanzar e insertarse y participar activamente en los mercados ampliados del departamento, el país y el continente.

Se logrará también, un cambio organizacional de fondo, el cual proporcionará efectividad en la gestión pública y privada provincial; contrarrestará los excesos de la corrupción y el clientelismo; fortalecerá la capacidad de aprendizaje y de respuesta territorial frente a la inestabilidad y la vulnerabilidad de la sociedad; e igualmente, desatará las posibilidades de cambio; abrirá nuevas ventanas de oportunidad y propiciará el desarrollo socioeconómico local.

\section{Lo que está en juego}

El reto es, entonces, convertir a la Provincia de Ubaté en un territorio organizado, que se convierta en plataforma sistémica propicia para la atracción de nuevas inversiones y, por consiguiente, para la creación de nueva riqueza incremental.

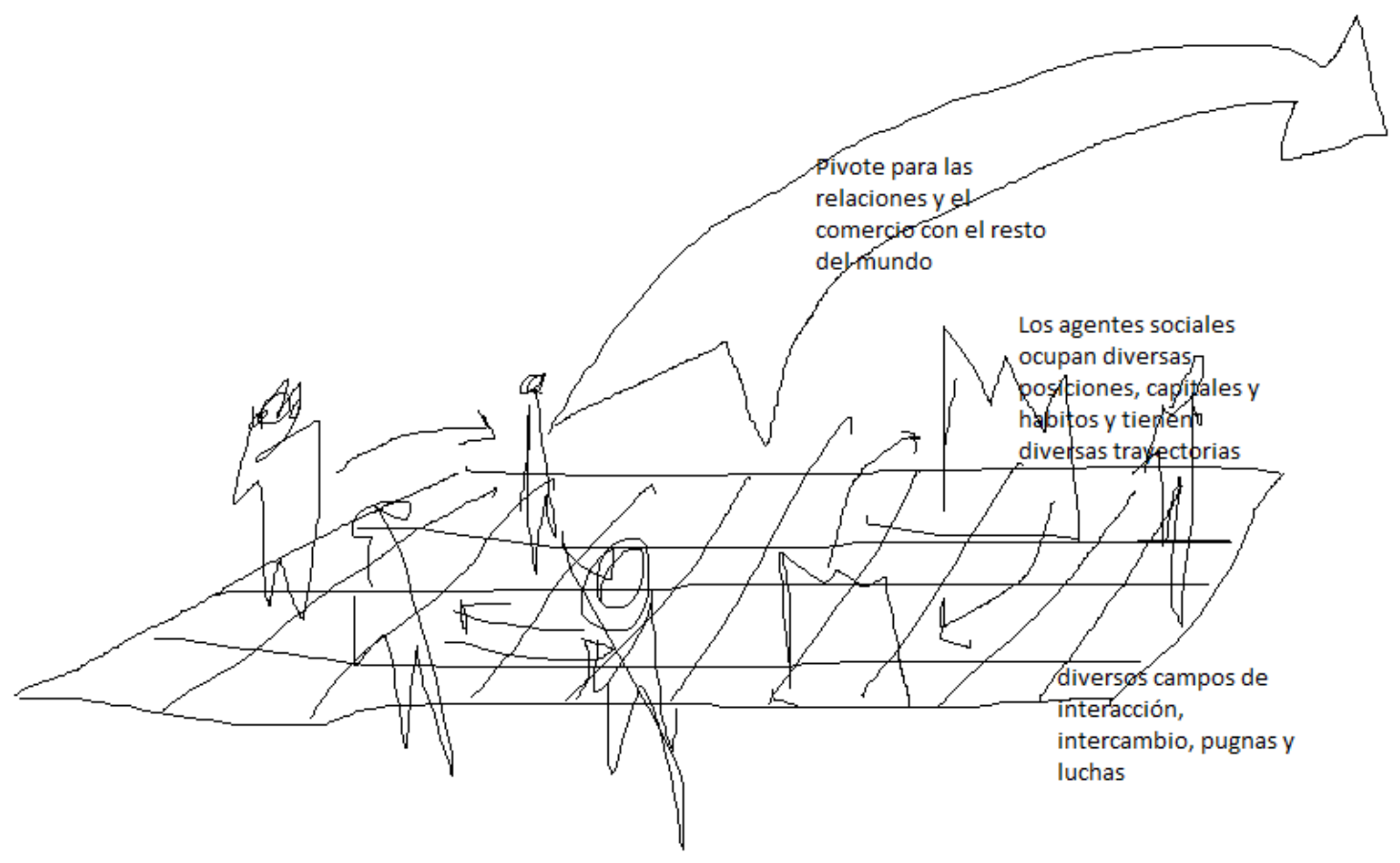

Las regiones organizadas se constituyen en plataforma sistémica para la atracción de inversiones, la competitividad y el desarrollo

FUENTE: diagrama diseñado y elaborado por Fernando L. Castellanos O. investigador del OBSOGED/LASO

Así, la inercia de la Provincia de Ubaté terminará rompiéndose positivamente, con la participación de grupos económicos y de nuevos inversionistas, de empresarios, del Gobierno, de la academia y de la sociedad civil; 
principalmente a través de los líderes locales, la fuerza intelectual y empresarial, los políticos, los dirigentes y la comunidad organizada a través de sus gremios y asociaciones.

\section{Conclusiones}

La búsqueda de objetivos ambiciosos estaría relacionada con la capacidad de asumir riesgos, como los asociados a la migración.

El flujo migratorio hacia Bogotá se ha visto acelerado por la urbanización, que acelera el "efecto demostración" y determina transformaciones irreversibles en la actitud de la gente de provincia.

El contacto con la sociedad urbana ha desarrollado en la gente la conciencia de las disparidades existentes entre el nivel de vida de Bogotá y el de los pueblos de provincia y zonas rurales, acuciadas por la subalimentación y pobres en asistencia médica y equipamiento escolar y oportunidades laborales.

Quiénes han pasado una temporada en Bogotá perciben que allí hay más amplias perspectivas de obtener un verdadero trabajo, estable y bien remunerado; que procura mejores oportunidades de acceder a estudio de calidad; y que procura un nivel de vida más confortable.

Muchas veces, la migración es más una solución que un problema, porque quien se marcha lo hace buscando oportunidades y una vida mejor. Pero la movilidad encierra, a su vez, un problema porque emigrar supone tensiones y dificultades. $\mathrm{Y}$ es que las personas que tienden a emigrar tienen índices mayores de insatisfacción en su vida, lo cual es lógico, si no, no se irían.

Las políticas de empleo sometidas al impacto de la mercantilización imperante que consideran el mercado como "estado de naturaleza", partiendo de la protección pública frente a la precariedad laboral y el desempleo, terminan por responsabilizar a los jóvenes de refugiarse en la dependencia familiar y estatal. Es así que proponen el emprendimiento como escudo de salvación.

La verdad es que en el sustrato de las políticas propuestas de emprendimiento hay varias falacias. En primer lugar, el mercado dejó de ser entendido como el problema (causante del desempleo) a partir del trabajo por cuenta propia que se entronizó como único merecedor del apoyo del Estado.

La protección colectiva de los derechos laborales se criminaliza como rigidez del mercado, para pasar a definir el empleo como una responsabilidad personal de cada individuo a partir de su iniciativa emprendedora. Con ello, el trabajo por cuenta ajena (empleo asalariado) queda devaluado como indigno de respaldo público.

¿Entonces por qué habría de venir ahora el Estado a respaldar iniciativas de emprendimiento sostenible para la Provincia de Ubaté? Esa es la gran pregunta, toda vez que si bien el desarrollo territorial pasa por el crecimiento económico que tales iniciativas implican y/o promueven, constituye, ante todo, un proceso socioeconómico, cultural, tecnológico, político y ambiental complejo; el cual, a no dudarlo, exige el reenfoque y la reorientación de los esfuerzos y de los recursos hacia un proyecto de desarrollo territorial compartido tanto por los ciudadanos como por los dirigentes del sector público y del sector privado de la Provincia. Y esto, porque el desarrollo territorial pasa también por la dotación de factores tanto naturales como creados, las cuales demandan cuantiosas inversiones.

Además, el desarrollo territorial está determinado por las relaciones de poder, los conflictos y la capacidad y diligencia decisoria de los dirigentes, en punto de las decisiones necesarias e inaplazables que deben asumir responsablemente y de su voluntad de llevar a cabo la gestión que potencie el territorio de la Provincia de Ubaté.

\section{Fuentes De Consulta}

Álvarez, S., \& Barney, J. (2014). Entrepreneurial Opportunities and Poverty Alleviation.Entrepreneurship Theory and Practice, 38(1), 1-26. DOI: 10.1111/ etap.12078

Barlocci, A. (2011) en el Prólogo a Kliksberg, B. (2011) Emprendedores Sociales, los que hacen la diferencia, Fundación Claritas, Argentina.

Bourdieu, P. y Sayad, A. (2017) El desarraigo. La violencia del capitalismo en una sociedad rural, Siglo XXI Editores, Buenos Aires, Argentina.

Broek, T., Ehrenhard, M., Langley, D., \& Groen, A. (2012). Dotcauses for sustainability: combining activism and entrepreneurship. Journal of Public Affairs, 12(3), 214-223. DOI:10.1002/pa.1435

Choi, D., \& Gray, E. (2008). The venture development processes of "sustainable" entrepreneurships. Management Research News, 31(8), pp.558-569. DOI: 10.1108/01409170810892127

Coase, R. (1960). El problema del coste social. En La empresa, el mercado y la ley. (p. 
223). Madrid: Alianza editorial.

Cohen, B., Smith, B., \& Mitchell, R. (2008). Toward a Sustainable Conceptualization of Dependent Variables in Entrepreneurship Research. Business Strategy andthe Environment, 17(2), 107-119. DOI:10.1002/bse.505

Cohen, B., \& Winn, M. (2007). Market imperfections, opportunity and sustainable entrepreneurship. Journal of Business Venturing, 22(1), 29-49. DOI: 10.1016/j. jbusvent.2004.12.001

Crals, E., \&Vereeck, L. (2005). The affordability of sustainable entrepreneurship certification for SMEs. International JournalofSustainableDevelopment\&WorldEcology, 12(2), 173-183. Doi: 10.1080/13504500509469628

Gil Villa, F (2016) La sociedad vulnerable, Tecnos, Madrid

Gómez D. (2015) "Dando voz al migrante que regresa", en Gutiérrez-Bonilla M. (editora) Identidades transnacionales. Jóvenes colombianos en contextos de migración internacional, Editorial Javeriana, Bogotá.

Norberto Elías What is Sociology? London, Hutchinson, 1978. La sociedad cortesana, Madrid, FCE, 1993.

Fernández Torres, A. "El análisis de entorno: un enfoque de planeación" Consultado en:www.uv.mx (Agostode 2019)

Nanus, B. (1982) “QUEST: Quick Environment Scanning Technique” En: Long Range Planning 15(2), p 39 - 45.

Neufeld, W.P. (1985) "Environmental Scanning: its use in Forecasting Emerging Needs and Issues in Organizations". En: Futures Research Quarterly 1(3) p $39-52$

Pacheco, D., Dean, T., \& Payne, D. (2010). Escaping the green prison: Entrepreneurship and the creation of opportunities for sustainable development. Journal ofBusiness Venturing, 25(5), 464-480. doi:10.1016/j.jbusvent.2009.07.006

Patzelt, H. \& (Shepherd, D. (2011). The New Field of Sustainable Entrepreneurship: Studying Entrepreneurial Action Linking "What Is to Be Sustained" With "What Is to Be Developed." EntrepreneurshipTheory and Practice, 35(1), 137-163. doi:10.1111/j.1540-6520.2010.00426.x

Pérez, A. (2017) La liberación del conocimiento. En El desarraigo. La violencia del capitalismo en una sociedad rural, Siglo XXI Editores, Buenos Aires, Argentina.

Ragin, Charles C. (1994) Constructing Social Research, Pine Forge Press.

Rodríguez, D. (2016). Emprendimiento sostenible, significado y dimensiones. Revista Katharsis, N. 21, pp.419-448. Recuperado de http://revistas.iue.edu.co/index.php/katharsis.

Sabogal, J. (2012) Entre la economía política de Karl Marx y la economía ecológica. Revista de Economía Institucional, 14(27), 207-222. Recuperado de http:// www.economiainstitucional.com/pdf/No. 27 pdf/jsabogal.pdf

Sayad, A. (2010) La doble ausencia. De las ilusiones del emigrado a los padecimientos del inmigrado, Editorial Anthropos, Barcelona.

Stiglitz, J. (2002) El malestar en la globalización (p.348). Buenos Aires: SantillanaEdiciones.

Tilley, F., \& Parrish, B. (2006) From poles to wholes: facilitating an integrated approach to sustainable entrepreneurship, Managemement and SustainableDevelopment, 2(4), 281-294. Recuperado de http://www.inderscience.com/link.php?id=10214

Tilley, F., \& Young, W. (2009). Sustainability Entrepreneurs: Could They Be the True Wealth Generators of the Future? Greener Management International, (55), 79-93. Recuperado de http://eprints.whiterose.ac.uk/77342/

Trigo y KaimowitzEconomía y sostenibilidad ¿pueden compartir el planeta? IICA, San José de Costa Rica, 1994. También alojado en FAO-CEDAF- Centro para el Desarrollo Agropecuario y Forestal, 1996.

Fuentes Institucionales

CCB-CEPEC/URosario Plan de Competitividad para la Provincia de Ubaté, Bogotá, 2013.

EXEDU (1991) Programa de Entrenamiento en Desarrollo Emprendedor para Docentes Universitarios, 1ra Promoción, Universidad de los Andes/Dow Chemical/Fundación Corona, Bogotá. Documentos varios.

ONU (1992) Declaración de Rio sobre el medio ambiente y el desarrollo. Rio de Janeiro. Recuperado de http://www19.iadb.org/intal/intalcdi/PE/2012/11109.pdf

\section{NOTAS}

${ }^{\mathrm{i}}$ Es importante subrayar que tanto los deseos como las creencias y las oportunidades de un individuo pueden verse afectados por, y afectar a, las acciones de otros individuos, dando lugar así a cadenas de influencia social que van configurando la vocación migratoria. Ahora, Sayad (2010) señala que "no se puede hacer sociología de la emigración sin esbozar, al mismo tiempo y de una vez, una sociología de la inmigración; pues la emigración aquí y la inmigración allá son las dos caras indisociables de una misma realidad, que no pueden explicarse la una sin la otra" en Sayad, Abdelmalek (2010) La doble ausencia. De las ilusiones del emigrado a los padecimientos del inmigrado, Editorial Anthropos, Barcelona. 
${ }^{\mathrm{ii}}$ Este sociólogo norteamericano concibe las configuraciones como "combinaciones de características o aspectos de los casos que los investigadores sociales contemplan en términos de aspectos comunes y diferencias" En Charles C. RaginConstructing Social Research, Pine ForgePress, 1994. Ya Norberto Elías había dicho que "el tejido social está atravesado por numerosas formas de interrelación que se entrecruzan como formas específicas de interdependencia que ligan unos individuos a otros, a las que llamo configuraciones, las cuales se caracterizan en general por la desigualdad, la dominación y el poder y que constriñen en distinta medida a los individuos. Ahora bien, sí al interior de ese tejido de interdependencias el individuo encuentra un margen de acción individual, al mismo tiempo le impone límites a su libertad de elección. De esta forma, el grado de autonomía (y por tanto de dependencia) de cada actor se debe determinar, en cada caso, mediante un análisis sociológico concreto" ver en Norberto Elías WhatisSociology? London, Hutchinson, 1978. También en Norberto Elías La sociedad cortesana, Madrid, FCE, 1993.

${ }^{i i i} S e$ está de acuerdo no sólo con Tilley y Young (2009) en que "la habilidad del emprendedor para reconocer y aprovechar oportunidades está condicionada por el contexto local y su estructura social" (Tilley\& Young, 2009) sino con Patzelt y Shepherd (2011) en que "aumenta con el conocimiento del medio natural y comunitario, de igual manera, su motivación para generar ganancias personales y para otros" (Patzelt\&Shepherd, 2011).

${ }^{\text {iv }}$ Se hace referencia aquí al estudio de los contextos que envuelven las acciones y decisiones políticas.

${ }^{\mathrm{v}}$ No sobra recalcar con Parra que cuando se habla de 'emprendimiento sostenible' se puede entender que se habla del emprendimiento como un motor de ideas y negocios transformadores del entorno desde la perspectiva de sostenibilidad para generar impactos positivos en términos de desarrollo social, económico y ambiental (Véase Parra, J.Emprendimiento sostenible, un motor para los retos de hoy, La República, Bogotá recuperado de https://www.larepublica.co/responsabilidad-social/emprendimiento-sostenible-un-motor-para-los-retos-de-hoy2049846 en Septiembre 6 de 2019.

${ }^{\mathrm{vi} J a m e s}$ Street concibe el desarrollo como "un proceso cultural complejo, en el que las fuerzas que inducen el crecimiento económico, como condición ineludible para alcanzar mejores condiciones de vida, continúan siendo el conocimiento y los instrumentos tecnológicos. Y las fuerzas que inhiben el progreso social están enraizadas en los patrones institucionales de comportamiento presentes en todas las sociedades, los cuales parecen ser particularmente obstructivos en las culturas de los países económicamente menos desarrollados" Véase Street J. "El desarrollo económico desde una perspectiva institucionalista" CEGA, Bogotá. Por otra parte, y desde otra perspectiva, el desarrollo de una sociedad territorial puede asimilarse a su "aptitud o capacidad efectiva para enfrentar con relativa eficacia los desafíos y problemas críticos que cada momento histórico le plantea: para satisfacer en forma tanto autogenerada como sostenida las necesidades materiales básicas y aspiraciones sociales de progreso individual y colectivo; así como para generar la riqueza necesaria para financiar el logro de dichos objetivos". Véase Amartya Sen Nuevo examen de la desigualdad, Alianza Editorial.

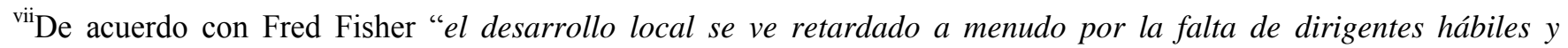
recursos y organizaciones ineficientes". Véase Fisher F. Administración del proceso de desarrollo, División de Desarrollo de Comunidades, Universidad del Estado de Pensilvania, EUA.

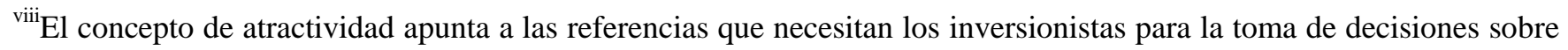
¿Dónde invertir? Y ¿En qué deben invertir productiva y rentablemente? Alude a "la capacidad para crear un ambiente territorial propicio para atraer inversión directa productiva hacia alli". Véase del WorldEconomicForum (1996) el informe sobre criterios e indicadores de atractividad territorial.

${ }^{\mathrm{ix}} \mathrm{La}$ creación de condiciones regionales competitivas se vuelve, entonces, cada vez más necesaria para generar el ambiente propicio a la formación de nuevas empresas y de cadenas productivas integradas. Tal proceso, indudablemente supone unas condiciones macroeconómicas favorables, pero también, la generación de las bases microeconómicas necesarias para estimular la toma de riesgos en un nuevo proyecto de sociedad y región capaz de atraer y estimular la inversión empresarial con énfasis en la elaboración de productos y el suministro de servicios de mayor sofisticación y valor agregado.

${ }^{x}$ En el entendido de que toda investigación parte, básicamente, de plantearse problemas que se derivan de lagunas, inconsistencias o deducción de consecuencias.

${ }^{x i}$ Proveniente del latín "contextus" el Diccionario de Uso delEspañol, de María Moliner, define el contexto como "el conjunto de circunstancias políticas, económicas, culturales, etc., que rodean un hecho" 


\begin{abstract}
${ }^{x i i}$ Según la Cámara de Comercio de Bogotá - CCB "esta situación contrasta notablemente con la registrada en provincias más cercanas a Bogotá como Soacha, Sabana Occidente y Sabana Centro, en las que el predominio de sectores económicos más urbanos (como el industrial y de servicios) ha favorecido una mayor concentración de población en las cabeceras municipales. En efecto, si se compara la densidad poblacional al interior de Cundinamarca, se puede apreciar cómo la Provincia de Ubaté cuenta con una tasa relativamente alta a nivel departamental (87 habitantes por kilómetro cuadrado), ubicándose por encima de provincias como Gualivá o Almeidas, lo que le permite posicionarse como el séptimo mercado del departamento. Sin embargo, si se tienen en cuenta los resultados de la Provincia de Soacha-Sibaté (que registra una densidad poblacional aproximadamente 19 veces superior), se corrobora el hecho de que la de Ubaté aún se perfila como una región con una orientación marcadamente rural" (CCBCEPEC/URosario Plan de Competitividad para la Provincia de Ubaté, Bogotá, 2013)

${ }^{\text {xiii }}$ Según Gil Villa nos dice "una sociedad puede denominarse vulnerable cuando amplios sectores de la población sufren riesgo de exclusión. La vulnerabilidad posee también un componente subjetivo que conecta con la tendencia a la expresividad de las emociones como rasgo de la cultura contemporánea. Una de las causas que hacen que la gente se sienta cada vez más vulnerable es la sensación de inseguridad" (Véase Gil Villa, Fernando La sociedad vulnerable, Tecnos, Madrid, 2016).
\end{abstract}

Tal como puede presumirse, esos sentimientos de inseguridad social e incertidumbre sobre el futuro que afecta a cada uno de nosotros, nos abre de par en par las puertas de lo que podemos llamar la "sociedad del miedo", cuyos fundamentos son la violencia simbólica y el miedo. Por una parte, la violencia estructural del desempleo y la precariedad laboral; $y$, por otro lado, el miedo al despido.

${ }^{x i v}$ En estos casos, la necesidad del presente, prima sobre cualquier previsión a largo plazo, sobre todo para aquellos campesinos y jornaleros agrícolas que disponen de pocos medios para adaptarse al mundo urbano y se ven obligados a aceptar empleos precarios e inestables, véase Pérez, 2017, Ibid.

${ }^{\mathrm{xv}}$ Es, tal como señala Gil Villa "juventud sin esperanzas, en una democracia concebida y soñada precisamente como medio fundamental para esparcir la dignidad entre todos los ciudadanos, pero ¿Qué sociedad puede alardear de haberlo conseguido, siquiera dentro de unos términos honorables, cuando su juventud navega como un barco, sin esperanza?" Véase Gil Villa, Fernando La sociedad vulnerable, Tecnos, Madrid, 2016, p. 33. 